\title{
PREVALÊNCIA DA DEFICIÊNCIA DE VITAMINA B12 EM PACIENTES DE UM LABORATÓRIO DE ANÁLISES CLÍNICAS DE FRANCISCO BELTRÃO, PR.
}

\author{
PREVALENCE OF VITAMIN B12 DEFICIENCY IN PATIENTS IN A \\ CLINICAL LABORATORY IN FRANCISCO BELTRÃO CITY, PR.
}

\author{
Dierings, L. R. ${ }^{1}$, Desidério, N. C. ${ }^{2}$, Mattiello, S. ${ }^{3}$, Foppa, D. P. ${ }^{4}$, Arruda, G ${ }^{5}$. \\ Afiliações: 1- Discente de Nutrição da Universidade Estadual do Oeste do Paraná- UNIOESTE. 2- Discente de Medicina da Pontifícia \\ Universidade Católica do Paraná. 3- Biomédica da Universidade Paranaense. 4- Discente de Farmácia da Universidade Paranaense. 5- \\ Docente da Universidade Estadual do Oeste do Paraná. \\ Rua Boa Vista, n² 29, Industrial, Francisco Beltrão, Paraná, Brasil. CEP: 85601-728. giselearrudabioq@ gmail.com
}

\section{Resumo}

Os compostos de cobalamina são designados como vitamina B12 e possuem um núcleo de corrina, que apresenta como elemento central o cobalto. Os alimentos fontes de vitamina B12 são carnes, leites e derivados e ovos, mas também podem ser sintetizados pelas bactérias pertencentes a microbiota intestinal, contudo não é fornecida ao hospedeiro em quantidades significativas. As enzimas que necessitam de vitamina B12 como cofator são a metionina sintase e $L$ metilmalonil-coA mutase, as quais estão envolvidas no metabolismo da homocisteína. A deficiência da vitamina B12 pode causar neuropatia periférica e sintomas psiquiátricos. $O$ objetivo deste trabalho foi estabelecer a prevalência da deficiência de vitamina $B 12$ em indivíduos que fizeram exame em um laboratório de análises clínicas de Francisco Beltrão, Paraná. Os dados foram obtidos diretamente do relatório de resultados de exames do laboratório. O valor médio de vitamina $B 12$ verificado foi de $243,7 \pm 101,1 \mathrm{pg} / \mathrm{mL}$ e $7,6 \%$ não estavam de acordo com os valores de referência. Já o valor médio de vitamina $D$ foi de $31,1 \pm 43,8 \mathrm{ng} / \mathrm{mL}$ e $21,4 \%$, encontravam-se fora dos valores de referência. Dos 85 idosos participantes, 3,5\% estavam abaixo e 7,0\% acima dos valores de referência para vitamina B12, mas entre os 47 idosos que realizaram exame de vitamina $D$, a prevalência de hipovitaminose foi de $70,2 \%$. Observa-se uma deficiência de vitaminas entre os pacientes deste estudo. Portanto, para evitar problemas relacionados à deficiência destas vitaminas e evitar diversos problemas de saúde, estas devem estar presentes em quantidade adequadas na alimentação, ou quando necessário a suplementação pode ser utilizada.

Palavras-chave: Cianocobalamina; Cobalamina; Complexo Vitamínico B.

\begin{abstract}
Cobalamin compounds are designated as vitamin B12 and have a corrin core, which has cobaltl as its central element. Food sources of vitamin B12 are meat, milk and dairy products and eggs, but it is also synthesized by bacteria belonging to the intestinal microbiotal, but is not supplied to the host in significant quantities. Enzymes that require vitamin B12 as cofactor, are methionine synthase and L-methylmalonyl-coA mutase, which are involved in homocysteine metabolism. Vitamin B12 deficiency can cause peripheral neuropathy and psychiatric symptoms. The objective was to establish the prevalence of vitamin B12 deficiency in individuals who were screened in a Francisco Beltrão, Pr clinical laboratory. Data were obtained. directly from the lab test results report. The mean value of vitamin $B 12$ was $243.7 \pm 101.1 \mathrm{pg} / \mathrm{mL}$ and $7.6 \%$ were not in accordance with the reference values. The mean vitamin $D$ value was $31.1 \pm 43.8 \mathrm{ng} / \mathrm{mL}$ and $21.4 \%$ were outside the reference values. Of the 85 elderly participants, $3.5 \%$ were below and $7.0 \%$ above the reference values for vitamin B12, but among the 47 elderly who underwent vitamin D examination, the prevalence of hypovitaminosis was $70.2 \%$. Therefore, to avoid problems related to the deficiency of these vitamins, they should be present in adequate amounts in the diet, or when necessary supplementation may be used.
\end{abstract}

Keywords: Cobalamin; Cyanocobalamin; Vitamin B Complex. 


\section{Introdução}

Os compostos de cobalamina são designados como vitamina B12 e possuem um núcleo de corrina, que apresenta como elemento central o cobalto ${ }^{1}$. Os alimentos fontes de vitamina B12 são carnes, leites e derivados e ovos, mas o que apresenta maior quantidade é o bife de fígado ${ }^{2}$.

A vitamina B12 é sintetizada pelas bactérias pertencentes a microbiota intestinal ${ }^{1}$, contudo não é fornecida ao hospedeiro em quantidades significativas ${ }^{3}$. As necessidades diárias variam conforme a faixa etária ${ }^{4}$.

As enzimas que necessitam de vitamina B12 como cofator são a metionina sintase e Lmetilmalonil-coA mutase, as quais estão envolvidas no metabolismo da homocisteína. A deficiência de vitamina B12, ocasiona um desvio metabólico, com formação de ácido metilmalônico e o aumento deste ácido no sangue e na urina, além do aumento de ácido propiônico, acarretando acidose metabólica. A inativação da enzima metilmalonil-coA mutase, contribui para o aparecimento de danos neurológico 5 .

$$
\text { O comprometimento da enzima }
$$
metionina sintase, responsável pela doação do grupo metil, leva ao acúmulo de 5-MTHF (5- metiltetraidrofolato e redução do folato disponível para formar 5,10metiltetrahidrofolaro, que atua como co-fator para síntese de DNA ${ }^{5}$. Causando consequências deletérias particularmente em células de divisão rápida ${ }^{1}$.

Uma das alternativas utilizadas como forma de reduzir os efeitos deletérios é fazer suplementação com ácido fólico ${ }^{1}$, impedindo aumento de HCY (hemocisteína) e consequente redução de lesões vasculares ${ }^{6}$. Assim como, redução no surgimento de alguns tumores ${ }^{7}$.

A deficiência da vitamina B12 pode causar neuropatia periférica e sintomas psiquiátricos, como transtorno depressivo e cognitivo. Baixos níveis sanguíneos de vitamina B12 são mais comuns em idosos, contudo, jovens e recém-nascidos podem $\operatorname{apresentar}^{8}$.

A vitamina B12 têm um papel importante no metabolismo e não pode ser sintetizada pelo organismo. Contudo, alguns pacientes podem não possuir uma alimentação adequada, ou apresentar fatores que impeçam sua metabolização, podendo, dessa forma, comprometer sua saúde. Sendo assim, o objetivo do presente trabalho foi estabelecer a prevalência da deficiência de vitamina B12 em 
indivíduos que fizeram exame em um laboratório de análises clínicas de Francisco Beltrão, Paraná.

\section{Métodos}

O presente estudo caracterizou-se como um delineamento observacional, quantitativo, sendo predominantemente uma pesquisa de campo, exploratória, quantitativa e retrospectiva. Os dados de níveis de vitamina B12, idade, sexo, estado fisiológico, uso de medicamentos e suplementação foram obtidos diretamente do relatório de resultados de exames do laboratório, dos primeiros seis meses do ano de 2019.

O projeto foi aprovado pelo Comitê de Ética em Pesquisa com Seres Humanos da Unioeste, através o parecer consubstanciado $\mathrm{n}^{\circ}$ 3.419.615.

\section{Resultados}

Nos primeiros meses do ano de 2019 foram realizados 225 exames de vitamina B12, destes $169(74,8 \%)$ eram de pacientes do sexo feminino e $57(25,2 \%)$ do sexo masculino. A idade média dos participantes da pesquisa foi de $50,3 \pm 43,8$ anos.

O valor médio de vitamina B12 verificado foi de $243,7 \pm 101,1 \mathrm{pg} / \mathrm{mL}$, sendo que o menor valor foi de $74 \mathrm{pg} / \mathrm{mL}$ e o maior foi superior a $1500 \mathrm{pg} / \mathrm{mL}$. Do total, $7,6 \%$ não estavam de acordo com os valores de referência (Tabela 1), os quais vão de 114 a 890 pg/mL para mulheres e 130 a 868 pg/mL para homens.

Tabela 1. Tabela referente ao resultado alterados de vitamina $\mathrm{B} 12$ e vitamina $\mathrm{D}$, em função da idade e sexo.

\begin{tabular}{|c|c|c|c|c|}
\hline & Sexo & $\mathrm{n}^{\circ}$ & VitB12* & VitD** \\
\hline Crianças & $\mathrm{M}$ & 3 & 01 & 00 \\
\hline $0-12$ & $\mathrm{~F}$ & 4 & 00 & 00 \\
\hline Adolescentes & M & 6 & 01 & 00 \\
\hline $13-21$ & $\mathrm{~F}$ & 10 & 00 & 00 \\
\hline Adultos & M & 26 & 01 & 00 \\
\hline $22-59$ & $\mathrm{~F}$ & 92 & 07 & 02 \\
\hline Idosos & M & 22 & 04 & 06 \\
\hline Acima 60 & $\mathrm{~F}$ & 63 & 03 & 27 \\
\hline $\begin{array}{l}* \mathrm{VitB} 12=\mathrm{n}^{\circ} \\
\text { vitamina } \mathrm{B} 12 ; \\
* * \mathrm{VitD}=\mathrm{n}^{\circ} \mathrm{de} \\
\text { D. }\end{array}$ & $\begin{array}{l}\text { cier } \\
\text { tes }\end{array}$ & & alores & $\operatorname{ados} d e$ \\
\hline
\end{tabular}

Já o valor médio de vitamina $\mathrm{D}$ foi de $31,1 \pm 43,8 \mathrm{ng} / \mathrm{mL}$ e do total dos exames realizados, 21,4\%, encontravam-se fora dos valores de referência, que são superiores a 20,0 $\mathrm{ng} / \mathrm{mL}$ para pacientes de até 60 anos e entre 30,0 e $60,0 \mathrm{ng} / \mathrm{mL}$ para pacientes do grupo de risco, que inclui pessoas acima de 60 anos. Contudo, nenhuma paciente apresentou risco de toxicidade e hipercalcemia, uma vez que, não houve valores acima de 100,0 ng/mL. 
Os valores médios dos resultados de vitamina B12, correlacionados com faixa etária e sexo, estão representados na Figura 1.

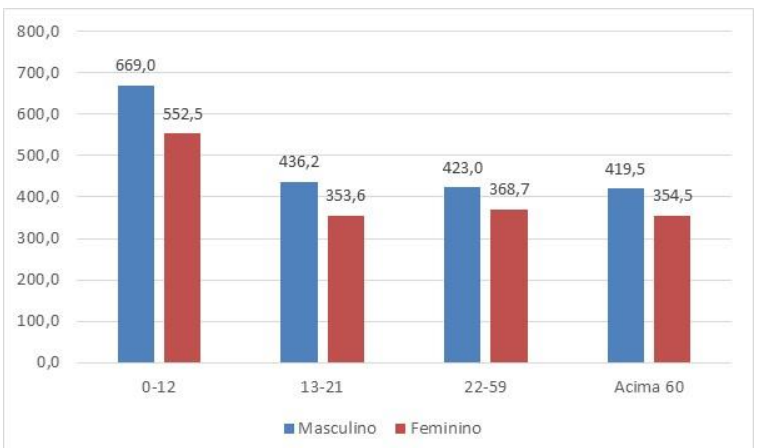

Figura 1. Valores médios de vitamina B12, em função da faixa etária e sexo.

Discussão

A análise dos resultados revelou que 118 pacientes adultos realizaram exames de Vitamina B12 e destes 2,4\% apresentaram valores abaixo da referência para vitamina B12 e 6,8\% para vitamina D. Já, dos 85 idosos participantes, 3,5\% tinham valores baixos e 7,0\% acima dos valores de referência para vitamina B12. No entanto, apenas 47 haviam realizado exame de vitamina $\mathrm{D}$ e neste grupo, a prevalência de hipovitaminose foi de 70,2\%.

Nazario e colaboradores verificaram que $32,8 \%$ dos pacientes, com diabetes mellitus tipo 2, em tratamento com metformina, entre 50 a 70 anos, apresentaram deficiência nos níveis séricos de vitamina B12 $(<200 \mathrm{pg} / \mathrm{mL})^{9}$. Contudo, outro estudo, mostrou que a deficiência bioquímica de vitamina B12 apresentava a incidência 7,4\%, em idosos ${ }^{10}$.
Valores estes superiores aos encontrados no presente estudo para idosos.

$\mathrm{O}$ valor médio de vitamina $\mathrm{B} 12 \mathrm{em}$ idosos foi $403,0 \pm 242,4 \mathrm{pg} / \mathrm{mL}$, valor este superior ao $337 \pm 191,2 \mathrm{ng} / \mathrm{L}$ encontrado por Bello e colaboradores, o qual, ainda elucidou que a população com déficit, apresentava idade mais avançada ${ }^{11}$, o que também pode ser verificado no presente estudo. Para crianças, o valor médio encontrado foi $602,4 \pm 192,5$ $\mathrm{pg} / \mathrm{mL}$, inferior ao $853,0 \mathrm{pg} / \mathrm{mL}$ verificado em um estudo realizado em Campinas ${ }^{12}$.

Alguns estudos demonstraram a associação entre declínio cognitivo, demência, doença de Alzheimer e níveis de vitamina B12 e levando em consideração o aumento contínuo da população idosa, a prevalência de demência estará aumentada ${ }^{13}$. Foi verificado em um estudo que $40 \%$ dos pacientes com níveis séricos reduzidos de vitamina B12, apresentaram afecções neurológicas não explicadas por causas vasculares ${ }^{14}$. Além de aumentar os níveis de homocisteína, que interfere nas ligações cruzadas de colágeno, diminuindo a resistência óssea, interferindo na osteoporose e em fraturas ósseas ${ }^{15}$. Da mesma forma que, a hipovitaminose $\mathrm{D}$, também é uma condição frequente associada ao risco 
aumentado de fraturas em idosos ${ }^{16}$, uma vez que, promove a reabsorção óssea de cálcio e absorção de cálcio e fósforo a partir do intestino, mantendo, portanto, os níveis de cálcio sérico ${ }^{17}$.

O valor médio de 25(OH)VD, em $\mathrm{ng} / \mathrm{mL}$, encontrado em um trabalho realizado em Belo Horizonte foi de $39,79 \pm 16,76^{18}$, com prevalência de hipovitaminose de $43,3 \%$, assim como Fernandes em 2016, também verificou uma prevalência de $48,4 \%^{19}$, valores estes divergentes dos revelados durante a investigação em Francisco Beltrão, Paraná, no qual a média foi de $31,1 \pm 43,8 \mathrm{ng} / \mathrm{mL}$, e a hipovitaminose esteve presente em 21,4\%. Contudo, diversos estudos mostraram a prevalência de hipovitaminose $\mathrm{D}$, sobretudo em $\operatorname{idosos}^{20}$.

\section{Conclusão}

A prevalência de vitamina B12 encontrada na população estudada foi menor do que a verificada em outros estudos, contudo foi possível verificar que entre os idosos a hipovitaminose é um pouco maior, o que pode acarretar problemas neurológicos. No entanto, a prevalência de hipovitaminose $\mathrm{D}$ neste grupo foi elevada, o que pode interferir no metabolismo ósseo, aumentando a probabilidade de problemas relacionados com osteoporose e fraturas ósseas.

$$
\text { Portanto, os cuidados com a }
$$

alimentação em relação aos micronutrientes, entre eles, vitamina B12 e D, devem receber atenção especial, principalmente para idosos, a fim de prevenir ou melhorar problemas relacionados a deficiência destas e quando necessário a suplementação pode ser utilizada.

\section{Referências}

${ }^{1}$ Mahan LK., Scott-stump S, Raymond JL. Krause: alimentos, nutrição e dietoterapia. Rio de Janeiro: Elsevier; 2018.

${ }^{2}$ Asbran. Tabela de conteúdo de vitamina b12 (cobalamina) nos alimentos. 2018. Associação Brasileira De Nutrologia. [acesso 30 mar. 2019] Disponível em: http://www.abran.org.br/images/agosto2010/vit amina\%20b12.pdf.

${ }^{3}$ Degnan PH, Taga ME, Goodman AL. Vitamin B12 as a modulator of gut microbial ecology. Cell Metab. 2014;20(5):769-778.

${ }^{4}$ Zago MA, Falcão RP, Pasquini R. Tratado de hematologia. São Paulo: Atheneu; 2018.

${ }^{5}$ Paniz C, Grotto D, Schmitt GC, Valentini J, Schott KL, Pomblum VJ, Garcia SC. Fisiopatologia da deficiência de vitamina B12 e seu diagnóstico laboratorial. J Bras Patol Med. 2005; 41(5):323-34.

${ }^{6}$ Food Ingredients Brasil. O ácido fólico e a saúde. 2016. [acesso 31 mar. 2018] Disponível em: http://revistafi.com.br/upload_arquivos/201607/2016070473 044001469734812.pdf.

${ }^{7}$ Baluz K, Carmo MdasGTDo, Rosas GO. Papel do ácido fólico na prevenção e na terapêutica oncológica: revisão. Rev. bras. cancerol. 2002; 48(4):597-607. 
${ }^{8}$ Martins JT, Carvalho-Silva M, Streck EL. Efeitos da deficiência de vitamina B12 no cérebro. Revista Inova Saúde. 2017; v. 6(1): 192-206.

${ }^{9}$ Nazário AR, Brittes KU, Haliski LB, Mizobuchi LS, Polonio RR. Prevalência da deficiência de vitamina B12 em indivíduos portadores de diabetes mellitus tipo 2 em uso de metformina. Rev Soc Bras Clin Med. 2018;16(2):99-103.

${ }^{10}$ Damião CP. Avaliação da frequência da deficiência de vitamina b12 nos pacientes diabéticos do tipo 2 em uso de metformina acompanhados no ambulatório de endocrinologia do HUAP-UFF. [dissertação]. Niterói: Universidade Federal Fluminense; 2015.

${ }^{11}$ Bello CT, Capitão RM, Duarte JS, Azinheira J, Vasconcelos C. Défice de vitamina B12 na Diabetes Mellitus Tipo 2. Acta Med Port. 2017;30(10):719-726.

${ }^{12}$ Barnabé A. Prevalência das deficiências de ácido fólico, vitamina B12 e ferro em diversos grupos da população brasileira, após o programa de fortificação adotado pela Anvisa. [tese]. Universidade Estadual de Campinas; 2014.

${ }^{13}$ Rodrigues CPC. Deficiência da vitamina B12 como um fator de risco na demência do idoso. [monografia]. Faculdade De Medicina Da Universidade De Coimbra; 2015

${ }^{14}$ Cassimiro JMM, Barreto Junior CA, Aguiar GPCGde, Oliveira OJNde. Deficiência de vitamina B12 em pacientes de uma enfermaria de clínica médica em Fortaleza/CE. Rev Med UFC. 2016;56(1):18-23.

${ }^{15}$ Coussirat C, Batista C, Schneider RH, Resende TdeL, Schwanke CH. Vitaminas B12, B6, B9 e homocisteína e sua relação com a massa óssea em idosos. Rev bras geriatr gerontol. 2012;15(3):577-585.

${ }^{16}$ Silva PZda, Schneider RH. O papel da vitamina $\mathrm{D}$ na força muscular em idosos. Acta Fisiatr. 2016;23(2):96-101.

${ }^{17}$ Pinheiro TMM. A Importância Clínica da Vitamina D. [dissertação]. Universidade Fernando Pessoa; 2015.

${ }^{18}$ Silva BCC, Camargos BM, Fujii JB, Dias EP, Soares MMS. Prevalência de Deficiência e Insuficiência de Vitamina D e sua Correlação com PTH, Marcadores de Remodelação Óssea e Densidade Mineral Óssea, em Pacientes Ambulatoriais. Arq Bras Endrocrinol Metab. 2008;52(3):482-488.

${ }^{19}$ Fermandes MG. Contribuições para diminuição da prevalência da deficiência de vitamina d em uma população assistida por uma equipe de saúde da família em Contagem/Mg. [monografia]. Universidade de Federal de Viçosa; 2016.

${ }^{20}$ Maeda SS, Borba VZC, Camargo MBR, Silva DMW, Borges JLC, Bandeira F, LazarettiCastro M. Recomendações da Sociedade Brasileira de Endocrinologia e Metabologia (SBEM) para o diagnóstico e tratamento da hipovitaminose D. Arq Bras Endocrinol Metab. 2014;58(5):411-433.

Data de submissão:_20/12//2019

Data de aprovação: 25/02/2020 